\title{
FAST 2-D DISCRETE COSINE TRANSFORM
}

\author{
MARTIN VETTERLI \\ ECOLE POLYTECHNIQUE FEDERALE DE LAUSANNE \\ 16 , Chemin de Bellerive \\ CH-1007 LAUSANNE, SWITZERLAND
}

\begin{abstract}
A fast radix-2 two dimensional discrete cosine transform (DCT) is presented. First. the mapping into a 2-D discrete Fourier transform (DFT) of a real signal is improved. Then an usual polynomial transform approach is used in order to map the 2-D DFT into a reduced size 2-D DFT and one dimensional odd DFT"s. Finally. optimized odd DFT algorithms for real signals are developped. Alltogether, a reduction of more than $50 \%$ in the number of multiplications and a comparable amount of additions is obtained in comparison to other algorithms.
\end{abstract}

\section{Introduction}

Block coding using the two dimensional discrete cosine transform is widely used in image data compression [1]. Usually, a small block (typically 8 by 8 or 16 by 16) is transformed and an appropriate bit allocation is made in the transform domain.

One approach to the 2-D DCT computation uses the separability property and computes a DCT of dimension $\mathrm{N}$ by $\mathrm{N}$ as 2N DCT's of $\mathrm{N}$ which can be computed by one of the known fast algorithms [2,3]. The other approach consists in computing directly the 2-D transform by means of polynomial transforms and was first proposed in $[4,5]$, restated in [6] and applied in [7]. Our method is also a direct approach to the 2-D problem. The DCT of $\mathrm{N}$ by $\mathrm{N}$ is mapped into a real DFT of $N$ by $N$ followed by post-multiplications. These post-multiplications are shown to be rotations, thus reducing the required number of operations. The real DFT is evaluated through polynomial transforms where the fact that the data is real is taken into account, specifically by developping an optimized real odd DFT algorithm.

\section{Evaluation of the DCT from a DFT}

First we consider the mapping from DCT to DFT and the optimization of the resulting post-multiplications. Assume a real signal $x\left(n_{1}, n_{2}\right)$ of dimension $N x N$. The 2-D DCT is defined as:

$$
\operatorname{DCT}\left(\mathbf{k}_{1}, \mathbf{k}_{2}, N\right)=\sum_{n_{1}=0}^{N-1} \sum_{n_{2}=0}^{N-1} x\left(n_{1}, n_{2}\right) \cdot \cos \left(\frac{2 \pi\left(2 n_{1}+1\right) k_{1}}{4 N}\right) \cdot \cos \left(\frac{2 \pi\left(2 n_{2}+1\right) k_{2}}{4 N}\right)
$$

In the following, we assume $\mathrm{N}$ to be a power of 2. Using the mapping introduced in [3] and then in [9] given by:

$$
\begin{aligned}
y\left(n_{1}, n_{2}\right) & =x\left(2 n_{1}, 2 n_{2}\right) \quad n_{1}=0 . N / 2-1, \quad n_{2}=0 . N / 2-1 \\
& =x\left(2 N-2 n_{1}-1,2 n_{2}\right) \quad n_{1}=N / 2-N-1, \quad n_{2}=0 . N / 2-1 \\
& =x\left(2 n_{1}, 2 N-2 n_{2}-1\right) \quad n_{1}=0 . N / 2-1, \quad n_{2}=N / 2 . N-1 \\
& =x\left(2 N-2 n_{1}-1,2 N-2 n_{2}-1\right) \quad n_{1}=N / 2 . N-1, \quad n_{2}=N / 2 . N-1
\end{aligned}
$$

The DCT becomes:

$$
\operatorname{DCT}\left(\mathrm{k}_{1}, \mathrm{k}_{2}, \mathrm{~N}\right)=\sum_{\mathrm{n}_{1}=0}^{\mathrm{N}-1} \sum_{\mathrm{n}_{2}=0}^{N-1} \mathrm{y}\left(\mathrm{n}_{1}, \mathrm{n}_{2}\right) \cdot \cos \left(\frac{2 \pi\left(4 \mathrm{n}_{1}+1\right) \mathrm{k}}{4 \mathrm{~N}}\right) \cdot \cos \left(\frac{2 \pi\left(4 \mathrm{n}_{2}+1\right) \mathrm{k}_{2}}{4 \mathrm{~N}}\right)
$$

Using basic trigonometry, this can be rewritten as phasors times real and imaginary part of a 2-D DFT. We use the following shorthands:

$$
\begin{aligned}
& \cos -\mathrm{DFT}\left(\mathrm{k}_{1}, \mathrm{k}_{2}, \mathrm{~N}\right)=\underset{\mathrm{n}_{1}=0}{\mathrm{~N}-1} \underset{\mathrm{n}_{2}=0}{\mathrm{~N}-1} \mathrm{y}\left(\mathrm{n}_{1}, \mathrm{n}_{2}\right) \cdot \cos \left(\frac{2 \pi\left(\mathrm{n}_{1} k_{1}+\mathrm{n}_{2} \mathrm{k}_{2}\right)}{\mathrm{N}}\right) \\
& \sin -\mathrm{DFT}\left(\mathrm{k}_{1}, \mathrm{k}_{2}, N\right)=\sum_{\mathrm{n}_{1}=0}^{N-1} \sum_{\mathrm{n}_{2}=0}^{N-1} y\left(\mathrm{n}_{1}, \mathrm{n}_{2}\right) \cdot \sin \left(\frac{2 \pi\left(\mathrm{n}_{1} \mathrm{k}_{1}+\mathrm{n}_{2} \mathrm{k}_{2}\right)}{\mathrm{N}}\right)
\end{aligned}
$$

which correspond to the real and imaginary part of a 2-D DFT. Equation (3) becomes:

$$
\begin{aligned}
\operatorname{DCT}\left(k_{1}, k_{2}, N\right)=1 / 2 & \cdot\left[\cos \left(\frac{2 \pi\left(k_{1}+k_{2}\right)}{4 N}\right) \cdot \cos -\operatorname{DFT}\left(k_{1}, k_{2}, N\right)\right. \\
& -\sin \left(\frac{2 \pi\left(k_{1}+k_{2}\right)}{4 N}\right) \cdot \sin -\operatorname{DFT}\left(k_{1}, k_{2}, N\right) \\
& +\cos \left(\frac{2 \pi\left(k_{1}-k_{2}\right)}{4 N}\right) \cdot \cos -\operatorname{DFT}\left(k_{1}, N-k_{2}, N\right) \\
& \left.-\sin \left(\frac{2 \pi\left(k_{1}-k_{2}\right)}{4 N}\right) \cdot \sin -\operatorname{DFT}\left(k_{1}, N-k_{2}, N\right)\right]
\end{aligned}
$$

Now, we note that DCT $\left(k_{1}, k_{2}, N\right)$, DCT $\left(N-k_{1}, k_{2}, N\right)$, DCT $\left(k_{1}, N-k_{2}, N\right)$ and $\operatorname{DCT}\left(\mathrm{N}-\mathrm{k}_{1}, \mathrm{~N}-\mathrm{k}_{2}, \mathrm{~N}\right)$ can be derived from the corresponding cos- and sin-DFT's by 2 plane rotations and some additions as follows:

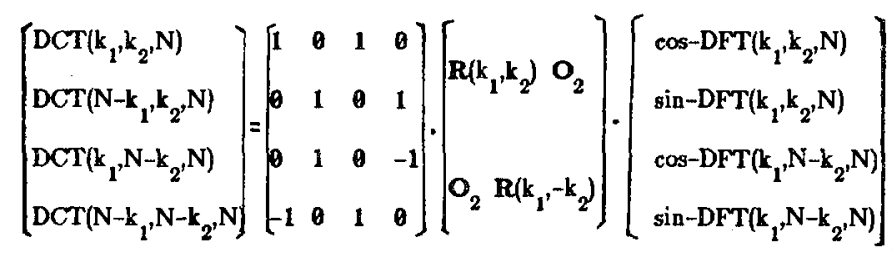

where:

$$
O_{2}=\left[\begin{array}{ll}
0 & 0 \\
0 & 0
\end{array}\right] \quad R\left(k_{1}, k_{2}\right)=\left[\begin{array}{cc}
2 \pi\left(k_{1}+k_{2}\right) & 2 \pi\left(k_{1}+k_{2}\right) \\
\cos \left(\frac{1 N}{4 N}\right) & -\sin \left(\frac{1 N}{4 N}\right) \\
\sin \left(\frac{2 \pi\left(k_{1}+k_{2}\right)}{4 N}\right) & \cos \left(\frac{2 \pi\left(k_{1}+k_{2}\right)}{4 N}\right)
\end{array}\right]
$$


Since $R\left(k_{1}, k_{2}\right)$ is a rotation matrix whose product with a 2-point vector requires 3 mults and 3 adds [9], the evaluation. of (6) requires a total 6 mults and 10 adds. Since (6) has to be evaluated for $k_{1}$ and $k_{2}$ going from 0 to $N / 2-1$, and taking into account all simplifications (eg. $k_{1}=k_{2}$ ), the load for obtaining the DCT from the real DFT is:

$3 \mathrm{~N}^{2} / 2-2 \mathrm{~N}$ mu. $\quad 5 \mathrm{~N}^{2} / 2-6 \mathrm{~N}+2$ ad.

While the development above is quite cumbersome, it should be noted that, especially for small transforms, the post-multiplications dominate the computational load. particularly for multiplies (Ex. 8x8 DCT: 24 multiplications for the real DFT and 80 for the post-multiplications)

\section{2-D Real DFT Calculation using Polynomial Transforms}

The real DFT will be computed using polynomial transforms in a way described in $[5,10]$ to which we refer for details. Below, the main steps are simply recalled. We want to evaluate:

$X\left(k_{1}, k_{2}\right)=\sum_{n_{1}=0}^{N-1} \sum_{n_{2}=0}^{N-1} x\left(n_{1}, n_{2}\right) \quad W^{z_{1} k_{1}} \quad W_{2}^{a_{2} k_{2}} \quad W=e^{-j 2 \pi / N}$

Where $N=2 m$. Since $x\left(n_{1}, n_{2}\right)$ is real, $X\left(k_{1}, k_{2}\right)$ is equal to $\left[X\left(N-k_{1}, N-k_{2}\right)\right]^{*}$. - denoting complex conjugates.

For $\mathbf{k}_{2}$ odd, equation (9) can be written as [5]

$X\left(k_{1}, k_{2}\right) \hat{=} X_{k_{1}}^{1}(z) \operatorname{Mod}\left(z-W^{k_{2}}\right) \quad k_{2}$ odd

where $x_{\mathbf{k}_{1}}^{1}(z) \cong \sum_{n_{1}=0}^{N-1} x_{n_{1}}^{1}(z) W^{a_{1} t_{1}} \operatorname{Mod}\left(z^{N / 2}+1\right)$

and $\quad x_{n_{1}}^{1}(z)=\sum_{n_{2}=0}^{N / 2-1}\left[x\left(n_{1}, n_{2}\right)-x\left(n_{1}, a_{2}+N / 2\right)\right] z^{n_{2}}$

since $\left(z-w^{k_{2}}\right), k_{2}$ being odd, is a factor of $\left(2^{N / 2}+1\right)$ which is in turn a factor of $\left(z^{N}-1\right)$. For $x\left(n_{1} n_{2}\right)$ real. (12) requires $N^{2 / 2}$ real additions. Now, $k_{1}$ in (11) is replaced by $k_{1} k_{2}$ (which is a valid permutation since $\mathrm{k}_{2}$ and $\mathrm{N}$ are relatively prime) Then, we can replace $W^{k} 2$ by $z$ in (11) since they are equivalent by (10). This leads to:

$x_{k_{1} k_{2}}^{1}(z) \triangleq \sum_{n_{1}=0}^{N-1} x_{n_{1}}^{1}(z) z^{n_{1} k_{1}} \quad \operatorname{Mod}\left(z^{N / 2}+1\right)$

This is a polynomial transform of length $N$ and a root $z$ defined modulo $\left(2^{\mathrm{N} / 2}+1\right)$ which can be computed with an FFT radix-2 type algorithm and uses $N^{2} / 2 \log _{2} N$ real additions for $X_{h_{1}}$ with real coefficients. Since $X_{k_{1 k}}(z)$ is of degree $N / 2-1$ and $k_{2}$ odd equal to $(2 u+1),(10)$ can be rewritten as:

$X\left(k_{1}(2 u+1),(2 u+1)\right)=\sum_{l=0}^{N / 2-1} y\left(k_{1}, l\right) w^{l} w^{2 u l}$

where $y\left(k_{1} l\right)$ is the 1 -th coefficient of the polynomial $x_{k_{110}}(z)$ Equation (14) represents $N$ add DFT's of length $N / 2$ whose computation will be adressed in the next section.

When both $k_{1}$ and $k_{2}$ are even, equation (9) reduces to a real DFT of size $N / 2$ by $N / 2$ on the real signal given by: $x\left(n_{1}, n_{2}\right)+x\left(n_{1}, n_{2}+N / 2\right)+x\left(n_{1}+N / 2, n_{2}\right)+x\left(n_{1}+N / 2, n_{2}+N / 2\right)$

When $k_{1}$ is odd and $k_{2}$ even, (9) can be rewritten, similarly to $(10-12)$, as

$X\left(k_{1}, 2 u_{1}\right) \Leftarrow X_{2 u k_{1}}^{1}(z) \operatorname{Mod}\left(z-W^{k_{1}}\right) \quad k_{1}$ odd

where $\quad x_{2 k_{1}}^{1}(z)=\sum_{D_{2}=0}^{N-1} x_{n_{2}}^{1}(z) z^{2 u n_{2}} \operatorname{Mod}\left(z^{N / 2}+1\right) \quad u=0 . N / 2-1$

and $\begin{aligned} & X_{n_{1}}^{1}(z)= \sum_{n_{1}=0}^{N / 2-1}\left[x\left(n_{1}, n_{2}\right)+x\left(n_{1}, n_{2}+N / 2\right)\right. \\ &\left.-x\left(n_{1}+N / 2, n_{2}\right)-x\left(n_{1}+N / 2, n_{2}+N / 2\right)\right] z^{n_{1}}\end{aligned}$

We note that (17) is a length $N / 2$ polynomial transform with a root $z^{2}$ defined modulo $\left(\mathrm{z}^{\mathrm{N} / 2}+1\right)$ which can be computed similarly to (13) using $\mathrm{N}^{2} / 2\left(\log _{2} \mathrm{~N}-1\right)$ real additions when $X\left(k_{1}, k_{2}\right)$ is real. Note that (15) and (18) require together $N^{2}$ additions. At last, (16) can be rewritten as (14), and thus evaluated as $\mathrm{N} / 2$ odd DFT's of length $\mathrm{N} / 2$.

All together, the real DFT of $\mathrm{N}$ by $\mathrm{N}$ has been mapped into $3 \mathrm{~N} / 2$ odd DFT's of length $\mathrm{N} / 2$ on real data and a real DFT of $\mathrm{N} / 2$ by $\mathrm{N} / 2$, at the cost of $3 \mathrm{~N}^{2} / 4 \log _{2} \mathrm{~N}+5 \mathrm{~N}^{2} / 4$ real additions.

\section{Computation of the real odd DFT's}

The length-N odd DFT's required for the evaluation of 2-D DFT's have the general form:

$X_{k}=\sum_{n=0}^{N-1} x(n) \quad W_{2 N}^{(2 k+1)} \quad W_{2 N}=e^{-j a \pi / 2 N}$

We introduce the following shorthands:

$\operatorname{\alpha cos-DFT}(k, n)=\sum_{n=0}^{N-1} x(n) \cos \left(\frac{2 \pi(2 k+1) n}{2 N}\right) \quad k=0 . . N-1$

$\operatorname{osin}-\operatorname{DFT}(k, n)=\sum_{n=0}^{N-1} x(n) \sin \left(\frac{2 \pi(2 k+1) n}{2 N}\right) \quad k=0 . N-1$

O-DCT $(k ; n)=\sum_{n=0}^{N-1} x(n) \cos \left(\frac{2 \pi(2 k+1)(2 n+1)}{8 N}\right) \quad k=0 . N-1$

and thus (19) is equal to:

$X_{k}=\operatorname{ocos}-D F T(k, N)-j \operatorname{osin}-D F T(k, N)$

Now we use a similar approach as in [9] to evaluate (23). The real part is reduced to:

$\cos -\operatorname{DFT}(\mathrm{k}, \mathrm{n})=\sum_{\mathrm{n}=0}^{N / 2-1} x(2 \mathrm{n}) \cos \left(\frac{2 \pi(2 \mathrm{k}+1) \mathrm{n}}{N}\right)$

$+\sum_{n=0}^{N / 4-1}(x(2 n+1)-x(N-2 n-1)) \cos \left(\frac{2 \pi(2 k+1)(2 n+1)}{2 N}\right)$ 
where we use the fact that $0-D C T(2 N-k-1, N)=-\sigma-D C T(k, N)$ Thus, with $N / 4$ input and $N / 2$ output additions, the odd $\cos -\mathrm{DFT}$ of $\mathrm{N}$ has been reduced to one of $\mathrm{N} / 2$ and an odd $\mathrm{DCT}$ of $\mathrm{N} / 4$. The imaginary part becomes:

$$
\begin{aligned}
& \operatorname{asin}-\operatorname{DFT}(k, n)=\sum_{n=0}^{N / 2-1} x(2 n) \sin \left(\frac{2 \pi(2 k+1) n}{N}\right) \\
& +\sum_{n=0}^{N / 4-1}(x(2 n+1)+x(N-2 n-1)) \sin \left(\frac{2 \pi(2 k+1)(2 n+1)}{2)}\right)
\end{aligned}
$$

Using trigonometric identities [9], this is equal to:

$$
\begin{aligned}
& \operatorname{osin}-\operatorname{DFT}(k, n)=\sum_{n \rightarrow 0}^{N / 2-1} x(2 n) \sin \left(\frac{2 \pi(2 k+1) n}{N}\right) \\
& +\sum_{n=0}^{N / 4-1}(-1)^{n}(x(2 n+1)+x(N-2 n-1)) \cos \left(\frac{2 \pi(N / 2-(2 k+1))(2 n+1)}{2 N}\right)
\end{aligned}
$$

or an odd $\sin -D F T$ of $N / 2$ and an odd DCT of $N / 4$ at the cost of $3 N / 4$ additions. Turning to the computation of the odd DCT, we use a mapping similar to [3]

$y(n)=x(2 n) \quad y(N-n-1)=-x(2 n+1)$

Thus, (22) becomes:

$0-D C T(k, n)=\sum_{n=0}^{N-1} x(n) \cos \left(\frac{2 \pi(4 k+1)(4 n+1)}{8 N}\right) \quad k=0 . . N-1$

Now, as seen in [9] o-DCT $(k, N)$ and o-DCT $(\mathrm{N}-\mathrm{k}-1, \mathrm{~N})$ are obtained from ocos-DFT(k,N) and osin-DFT(k,N) by a simple rotation or 3 multiplications and 3 additions.

Evaluating the computational complexity of this approach to the odd DFT computation of length $\mathrm{N}$ real signals, it is seen that it requires:

$\mathrm{N} / 2\left(\log _{2} \mathrm{~N}-1\right) \mathrm{mu} . \quad 3 \mathrm{~N} / 2\left(\log _{2} \mathrm{~N}-1\right)$ ad.

Note that when this real odd DFT algorithm is used for complex signals (by transforming separately the real and imaginary part and adding the result) it leads to the same number of multiplies as the Rader/Brenner FFT but to substantially iess additions.

\section{Complexity evaluation and comparison}

Using the above introduced odd DFT algorithm leads to the following load for a real DFT of $\mathrm{N}$ by $\mathrm{N}, \mathrm{N}$ a power of 2:

$\mathrm{N}^{2} / 2 \log _{2} \mathrm{~N}-7 / 6 \mathrm{~N}^{2}+4 \mathrm{mu}$

$5 N^{2} / 2 \log _{2} N-13 / 6 N^{2}+56 / 3$ ad.

and, with the optimized mapping for the DCT, the complexity for a real DCT of $\mathrm{N}$ by $\mathrm{N}$ is:

$\mathrm{N}^{2} / 2 \log _{2} \mathrm{~N}-2 \mathrm{~N}+\mathrm{N}^{2} / 3+8 / 3 \mathrm{mu}$

$5 N^{2} / 2 \log _{2} N-6 N+N^{2} / 3+62 / 3$ ad.

These results compare favorably with existing algorithms. For completeness, we compare it with: a) The Chen et al. algorithm for a row/column approach with a 1-D DCT that uses:

$\mathrm{N} \log _{2} \mathrm{~N}-3 / 2 \mathrm{~N}+4 \mathrm{mu} . \quad 3 / 2 \mathrm{~N} \log _{2} \mathrm{~N}-3 / 2 \mathrm{~N}+2$ ad.

b) The polynomial approach from [7] which is slighty more efficient then the one in [6].

c) A row/column approach with the 1-D DCT algorithm from [9] which uses:

$\mathrm{N} / 2 \log _{2} \mathrm{~N} \mathrm{mu} . \quad \mathrm{N} / 2\left(3 \log _{2} \mathrm{~N}-2\right)+1$ ad.

d) The proposed method.

The complexities are compared in table 1 and operation counts are given in table 2 . Asymptotically, it reduces the number of multiplies by a factor of 4 and saves $1 / 6$ of the adds when compared to currently proposed algorithms (a,b above).

\section{Implementation considerations}

The above algorithm, while achieving substancial computational savings, has a rather involved structure. But for the expected application (image coding with -blocks of $8 \times 8$ or $16 \times 16$ ), the transform can be explicitely written in linear code [11] Therefore, the structural complexity dissappears completely.

Furthermore, using signal processors or specialized processors with a large number of registers (for ex. TMS 320 with 144 registers) allows to perform the whole transform in the registers, thus avoiding the data transfer problem during the transform evaluation. This should lead to fast implementations where the computational savings are fully translated into time savings.

\section{Conclusion}

A fast 2-D DCT algorithm was proposed which reduces the number of multiplies by 50 to $75 \%$ with comparable number of additions.

This was achieved by showing that a 2-D DCT can be obtained from a real 2-D DFT with 1.5 multiplies per point and by developping efficient real odd DFT algorithms which are used when a 2-D DFT is evaluated through polynomial transforms.

\section{Acknowledgemente}

The author wishes to thank Prof. H.J. Nussbaumer for commenting the manuscript, the "Fonds National Suisse de la Recherche Scientifique" for supporting this research and Michael Unser for invaluable help.

\section{References:}

[1] A.K. Jain, "Image Data Compression : A Review", Proceedings of the IEEE, Vol.69, No.3, March 1981, pp. $349-389$.

[2] W-H. Chen, C.H. Smith, and S.C. Fralick, "A Fast Computational Algorithm for the Discrete Cosine Transform", IEEE Trans. on Communications, Vol. COM-25, pp.1004-1009, Sept. 1977.

[3] M.J. Narasimha, and A.M. Peterson, "On the Computation of the Discrete Cosine Transform". IEEE Trans. on Communications, Vol. COM-26. pp. 934-936, June 1978 
[4] H.J. Nussbaumer, "Fast Polynomial Transform Computation of the 2-D DCI". Proc. of the Int. Conf. on Digital Signal Processing, pp. 276-283, Florence, 1981.

[5] H.J. Nussbaumer, Fast Fourier Transform and Convolution Algorithms, 2nd. ed., Springer, Berlin, 1982.

[6] N. Nasrabadi, and R. King. "Computationally Efficient Discrete Cosine Transform Algorithm", Elec. Letters, 6th Jan. 1983, Vol.19, No. 1. pp. 24-25.

[7] S-C. Pei, and E-F. Huang, "Improved 2D Discrete Cosine Transforms Using Generalized Polynomial Transforms and DFT's", Proc. IEEE Int. Conf. on Communications, Amsterdam, 1984, pp. 242-244.

[8] J. Makhoul, "A Fast Cosine Transform in One and Two Dimensions", IEEE Trans. on ASSP, Vol. ASSP-28, No. 1. Feb. 1980, pp. 27-34.
[9] M. Vetterli, and H.J. Nussbaumer, "Simple FFT and DCT Algorithms with Reduced Number of Operations", Signal Processing, Vol. 6, No. 4, July 1984.

[10] H.J. Nussbaumer, and P. Quandalle, "Fast Computation of Discrete Fourier Transforms Using Polynomial Transforms". IEEE Trans. on ASSP, Vol. ASSP-27, pp. 169-181, 1979.

[11] L.R. Morris, "Automatic Generation of Time Efficient Digital Signal Processing Software ". IEEE Trans. Acoust. Speech, Signal Processing, Vol. ASSP-25, pp.74-78, Feb. 1977.

TABLE I: Computational complexity for the algorithms a)-d)

Algorithm Multiplications

a)

b)

c)

d)
$2 \mathrm{~N}^{2} \log _{2} \mathrm{~N}-3 \mathrm{~N}^{2}+8 \mathrm{~N}$

$2 \mathrm{~N}^{2} \log _{2} \mathrm{~N}$

$\mathrm{N}^{2} \log _{2} \mathrm{~N}$

$\frac{\mathrm{N}^{2}}{2} \log _{2} \mathrm{~N}+\frac{\mathrm{N}^{2}}{3} 2 \mathrm{~N}+\frac{8}{3}$
Additions

$$
\begin{aligned}
& 3 N^{2} \log _{2} N-3 N^{2}+4 N \\
& 5 N^{2} \log _{2} N-2 N^{2} \\
& 3 N^{2} \log _{2} N-2 N^{2}+2 N \\
& \frac{5 N^{2}}{2} \log _{2}+\frac{N^{2}}{3}-6 N+\frac{62}{3}
\end{aligned}
$$

TABLE II: Operation counts for the algorithms a)-d)

a)

\begin{tabular}{rrrrrrrrrr}
$N$ & mults & adds & mults & \multicolumn{1}{l}{ adds } & \multicolumn{1}{l}{ mults } & adds & mults & adds \\
8 & 256 & 416 & 384 & 832 & 192 & 464 & 104 & 474 \\
16 & 1408 & 2368 & 2048 & 4608 & 1024 & 2592 & 568 & 2570 \\
32 & 7424 & 12416 & 10240 & 23552 & 5120 & 13376 & 2840 & 12970 \\
64 & 37376 & 61696 & 49152 & 114688 & 24576 & 65664 & 13528 & 62442 \\
128 & 181248 & 295424 & 229376 & 540672 & 114688 & 311552 & 62552 & 291434 \\
256 & 854016 & 1377280 & 1048576 & 2490368 & 524288 & 1442304 & 283480 & 133105
\end{tabular}

